\title{
Nilotinib related acute myocardial infarction with nonobstructive coronary arteries: a case report and literature review
}

\author{
Weiwei Chen ${ }^{1,2}$, Beibei Du ${ }^{1,2^{*}}\left(\mathbb{D}\right.$, Kun Liu $^{1,2}$, Zhixi Yu ${ }^{1,2}$, Xingtong Wang ${ }^{3}$ and Ping Yang ${ }^{1,2}$
}

\begin{abstract}
Background: Myocardial Ischemia with No Obstructive Coronary Artery Disease (MINOCA) is a common cause of type 2 acute myocardial infarction (AMI) which requires careful differential diagnosis. Coronary artery spasm (CAS) syndrome is one etiology that can lead to MINOCA. Nilotinib, a targeted treatment for chronic myeloid leukemia (CML), has been reported to be related with increased risk of adverse vascular events.

Case presentation: A 67-year-old male patient was admitted to hospital with acute chest pain. He had a past medical history of CML and a history of treatment with nilotinib for 12 months. Coronary angiography (CAG) showed no significant stenosis. Since the onset of angina was generally in the early morning, and ECG and echocardiography suggested right coronary artery (RCA) disease, an ergonovine provocation test was performed to confirm the diagnosis of CAS. After intracoronary administration of ergonovine, middle and distal RCA showed over 90\% vasoconstriction. Nilotinib related MINOCA, CAS and CML were diagnosed. Lifestyle changes (cessation of smoking), anti-spasmodics, statin treatment and adjustment of the nilotinib dose (from $200 \mathrm{mg}$ bid, to $150 \mathrm{mg}$ bid) were recommended for this patient. Six-month's follow-up showed good recovery with no onsets of angina.
\end{abstract}

Conclusions: Physicians should be vigilant to adverse vascular events when treating patients who have been prescribed nilotinib. It is suggested that in patients with MINOCA who have a history of treatment with nilotinib, CASinduced MINOCA should be included in the differential diagnosis. Further studies are needed to clarify the mechanism and to find better management.

Keywords: Myocardial Ischemia with No Obstructive Coronary Artery Disease, Coronary artery spasm, Nilotinib, Ergonovine provocation test, Vascular adverse events, Case report

\section{Background}

The increased understanding of acute myocardial infarction (AMI) has led to the need for a general, worldwide definition. The European Society of Cardiology (ESC), the American College of Cardiology (ACC) and the American Heart Association (AHA), together with the World

\footnotetext{
*Correspondence: beibeidu2012@jlu.edu.cn

${ }^{1}$ Department of Cardiology, China-Japan Union Hospital of Jilin

University, Xiantai, Street No. 126, Changchun 130033, Jilin Province,

China

Full list of author information is available at the end of the article
}

Heart Federation (WHF), brought out the Fourth Universal Definition of myocardial infarction (MI) in 2018, and reclassified AMI into 5 subtypes[1]. Any ischemic myocardial injury that is caused by the imbalance of oxygen supply and oxygen need was classified as type 2 MI [1]. Myocardial ischemia with no obstructive coronary artery disease (MINOCA) commonly leads to type 2 MI which needs careful differential diagnosis [2]. The diagnosis of MINOCA should be made in the absence of obstructive (lesion $\geq 50 \%$ ) coronary artery disease. Coronary artery spasm (CAS) syndrome, coronary dissection, in situ thrombosis [3], tachycardia, and coronary embolism [4] 
are all etiologies that can cause MINOCA. The careful collection of medical history, and specialized examinations such as intracoronary imaging or the ergonovine provocation test, can facilitate the differential diagnosis.

Nilotinib, a second-generation BCR-ABL tyrosine kinase inhibitor (TKI), has greatly improved the treatment and prognosis of chronic myeloid leukemia (CML) [5]. Adverse vascular events (including angina, type $1 \mathrm{MI}$, and peripheral artery diseases) have been reported in patients treated with nilotinib [6,7]. Here we report the diagnosis and treatment of a rare case of nilotinib related MINOCA.

\section{Case presentation}

A 67-year-old male patient was admitted to China-Japan Union hospital for persistent chest pain two days before admission in Aug. 2020. The patient's chest pain started 2 days previously with no inducement, and was gradually alleviated $30 \mathrm{~min}$ after oral nitroglycerin intake. Over the next two days before he was referred to hospital, he had onsets of angina in the early mornings. The patient was diagnosed with CML 6 years previously. The patient was initially treated with imatinib, which was then changed to nilotinib (300 mg bid) in Dec 2019. A cytogenetic and molecular response was achieved in Mar 2020. The patient had no history of hypertension or diabetes, but had a 45-pack-year smoking history. No abnormalities were found during physical examinations.

Cardiac injury biomarkers showed significantly increased levels of troponin I $(4.4 \mathrm{ng} / \mathrm{ml}$; normal range 0-0.05), myoglobin (181 mg/L; normal range 0-107), and CK-MB (49.0 U/L; normal range 0-4.3). D-dimer and NT-proBNP levels were slightly increased. LDL-C level was $3.41 \mathrm{mmol} / \mathrm{L}$, and blood sugar level was normal. Other laboratory tests were within the normal range (Detailed information see Table 1).

Electrocardiography (ECG) upon admission showed pathological Q waves and slightly elevated ST segments in Leads III and aVF. T wave inversions were also found in precordial leads (Fig. 1A). ECG showed inferior wall hypokinesis and normal cardiac function (EF $60 \%)$. Holter monitoring for $24 \mathrm{~h}$ showed no ventricular arrhythmias (VAs). Color doppler ultrasound of carotid and subclavian arteries were normal. Coronary angiography (CAG) was performed and showed approximately $30 \%-40 \%$ stenosis in the proximal left anterior descending artery (pLAD) and the proximal obtuse marginal artery (pOM) (Figs. 1B, C). There was only mild to moderate stenosis (30-40\%) in the anticipated culprit vessel right coronary artery (RCA; Fig. 1D, E). Since there had been frequent onsets of angina in the early mornings, and also no significant or suspicious lesions were found during CAG, coronary spasm
Table 1 Laboratory test results of this patient

\begin{tabular}{ll}
\hline Category & Value [Normal range] \\
\hline White blood cell $\left(* 10^{9} / \mathrm{L}\right)$ & $8.4[4-10]$ \\
Neut $(\%)$ & $80 \uparrow[50-70]$ \\
Troponin I $(\mathrm{ng} / \mathrm{ml})$ & $4.4 \uparrow[0-0.05]$ \\
Myoglobin $(\mathrm{mg} / \mathrm{L})$ & $181 \uparrow[0-107]$ \\
CK-MB $(\mathrm{U} / \mathrm{L})$ & $49.0 \uparrow[0-4.3]$ \\
D-dimer $(\mathrm{ng} / \mathrm{ml})$ & $837 \uparrow[0-600]$ \\
NT-pro BNP & $540 \uparrow[300-450]$ \\
LDL-C (mmol/L) & $3.41 \uparrow[<1.8]$ \\
Other tests & Normal \\
\hline
\end{tabular}

was considered the etiology. An ergonovine provocation test was performed to confirm the diagnosis of CAS. After intracoronary administration of ergonovine $(0.04 \mathrm{mg})$, the middle and distal segments of RCA showed diffuse coronary spasm (Fig. 2A, B) compared to the baseline CAG of RCA (Fig. 1D, E). 5 min after ergonovine was administered, CAG showed near subtotal occlusion in middle and distal RCA. Continuous ECG monitoring showed more prominent ST elevations in inferior wall leads (Fig. 2A', B'). The patient also had chest discomfort after ergonovine administration, which later developed into chest pain at $5 \mathrm{~min}$. The ECG showed dynamic changes, which were all rapidly relieved after intracoronary administration of nitroglycerin (Fig. 2C, C').

According to the medical history, the CAG findings, and the ergonovine provocation test, the patient was diagnosed with Nilotinib related MINOCA, Killip class I, CAS syndrome, and CML.

Lifestyle changes were recommended including the cessation of smoking. Stenting was not recommended as there was no severe stenosis and after ergonovine administration diffuse spasm were observed. Intensified anti-spasmodics were recommended for long-term management with Isoket ampoule injection $(10 \mathrm{mg} / \mathrm{h}$ IV) and Diltiazem Sustained Release Tablets (90 mg bid). Statins were also prescribed to maintain the stability of the plaque and to protect the endothelial function. After hematologist consultation, the nilotinib dose was adjusted to $150 \mathrm{mg}$ bid.

The chest pain was completely relieved by the above treatments. The only adjustment to the treatment schedule occurred five days later with the replacement of the Isoket ampoule injection with Isoladine $50 \mathrm{mg}$ once daily (QD). The patient was discharged one week after the procedure. Follow-ups were conducted every three months, and after six months there was still good recovery with no onsets of angina. Also during 


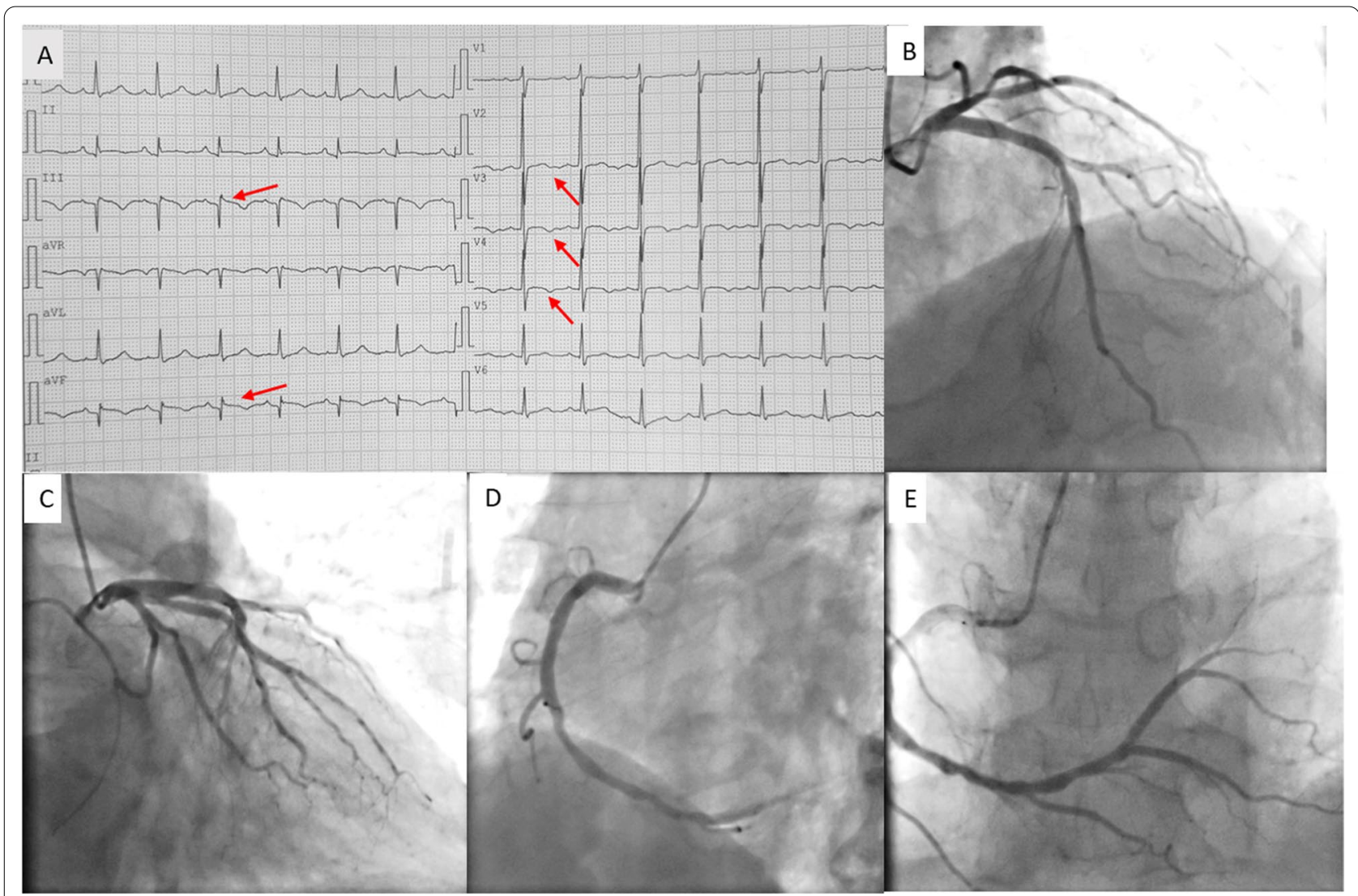

Fig. 1 ECG upon admission, and Baseline CAG. A Admission ECG: Leads III and aVF: pathological Q waves, slightly elevated ST segments. Precordial leads: T wave inversions. Red arrows indicate abnormalities in ECG. B-E. Baseline CAG. CAG showed 30-40\% stenosis in pLAD (B) and pOM (C) 30-40\%stenosis in dRCA (D, E). ECG: electrocardiography, CAG: coronary angiography, pLAD: proximal left anterior descending artery, pOM: proximal obtuse marginal artery, dRCA: distal right coronary artery

follow-ups, no peripheral artery rapid progression or new cerebrovascular disease were found.

\section{Discussion}

Here we presented a rare case of nilotinib related MINOCA, and further confirmed that CAS was the etiology of MINOCA, using an ergonovine provocation test. Symptom relief and recovery were achieved by lifestyle changes, anti-spasmodic medications, and adjustment of the nilotinib dose.

MINOCA has been shown to account for $5-15 \%$ of AMI cases [2, 8]. Furthermore, as a "working" but not a final diagnosis, screening for the underlying etiology of MINOCA is vital for subsequent proper management. Careful collection of medical history and specialized examinations, are required in the differential diagnosis [8]. For this patient, the history of nilotinib treatment is important in the exploration of the underlying etiology.

BCR-ABL tyrosine kinase inhibitors (TKIs) have shifted the treatment paradigm, and significantly improved the prognosis of chronic myeloid leukemia
(CML). However, in recent years, vasculotoxicity or vascular adverse events (VAE) (including peripheral arteries and coronary artery) have become a concern in patients receiving such treatment [9]. Although not fully characterized, based on three retrospective studies [9-11], patients who developed TKI-related VAE commonly had their first VAE onset within the first year. For this patient, he started the medication (nilotinib) since Dec. 2019, and had AMI 9 months (Aug. 2020) afterward. This is also consistent to the results of the retrospective studies.

Compared to the first generation BCR-ABL TKI (imatinib), the risk of VAE with nilotinib (second generation BCR-ABL TKI) was over threefold higher [11]. In one pooled analysis of three prospective clinical studies, during a quite long follow-up of 77 months in 108 patients who were treated with nilotinib, the incidence of angina/MI was 6\% (7/108) [10]. Apart from AMI, other potent VAE such as cerebrovascular or peripheral artery rapid progression, which manifested as intermittent claudication or stroke, have also been reported [7, 12, 13]. 


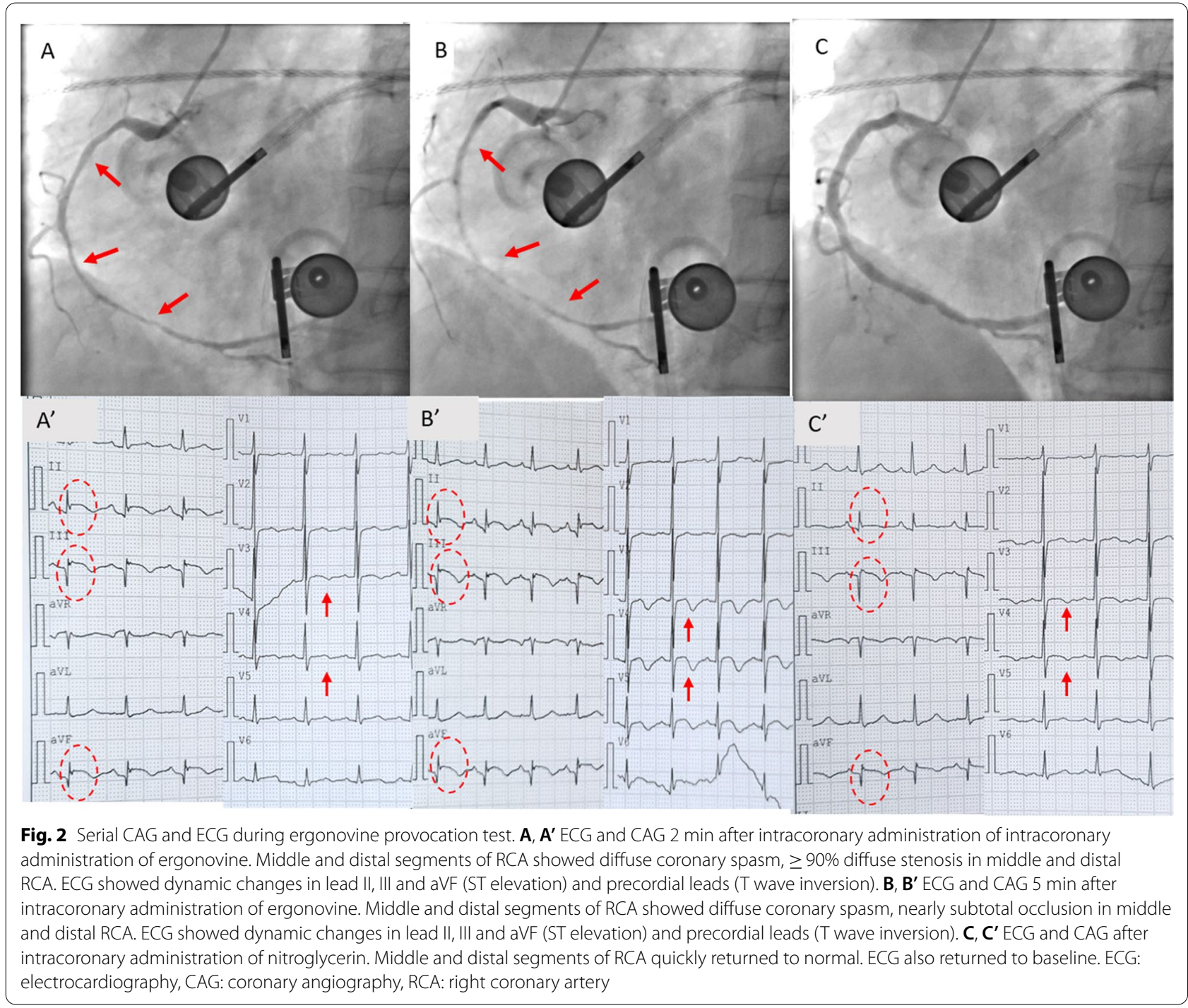

Still, the diagnosis is mainly made based on medical history and clinical experience.

The mechanism of nilotinib related cardiovascular disease is still unclear. Lipid metabolism disorder [14], endothelial dysfunction [9] and inflammation overactivation [15] are several suggested mechanisms, which can accelerate atherosclerotic process and cause vascular spasm [16]. To the best of our knowledge, there have been only three cases reported of nilotinib related coronary spasm $[17,18]$. No CAS induced MINOCA related to nilotinib has been previously reported.

Patients with traditional cardiovascular $(\mathrm{CV})$ risk factors, such as old age ( $\geq 60$ years), smoking, or high cholesterol level, were at higher risk of developing vasculotoxicities [19]. Therefore, baseline systematic coronary risk evaluation (SCORE) assessments and baseline CML-CV scores were developed to enable the prediction of adverse CV events [20]. For this patient, advanced age, smoking and high cholesterol level are all risk factors in developing vasculotoxicity. So, during the CAG, atherosclerosis and coronary spasm should also be assessed.

Focal or diffuse coronary spasm can show concomitant changes with plaque rupture/erosion in obstructive AMI patients (Type I AMI). CAS can be a common cause of MINOCA and an ergonovine/ acetylcholine provocation test is the gold standard for the diagnosis of CAS. An ergonovine provocation test was implemented by evaluating the response of intracoronary administration of ergonovine/ acetylcholine by CAG. CAS was defined as $>90 \%$ vasoconstriction of an epicardial coronary artery and compromised coronary blood flow. Although ergonovine/ acetylcholine provocation test is considered the gold standard for diagnosis, it is not routinely used in most catheter labs in China due to safety concerns; this 
can lead to misdiagnosis and under realization of CAS. In one study, the percentage of CAS diagnosed with the positive provocation test was $46.2 \%(37 / 80)$ and also in this study, good safety data with no major adverse events were reported [21].

The manifestations of CAS can vary vastly from being asymptomatic to angina, AMI, ventricular arrhythmias (VAs), or even syncope, with different coronary segments involved and different frequencies of angina onsets [22]. The treatments of CAS mainly involves lifestyle changes (such as cessation of smoking), anti-spasmodic medications, and other cardiovascular protective therapy [22]. Stenting for diffuse coronary spasm is not recommended, but in patients with focal spasm, its utility is unclear [22-24]. In patients with frequent onsets of angina, risk stratification is needed and for high-risk patients with life-threatening VAs, ICD therapy is recommended for secondary prevention [24]. For this patient, with above optimized medical treatment, symptom relief and good recovery were also achieved.

Optimal management of nilotinib related CAD needs the cooperation and teamwork of cardiologists and hematologists, especially in those with life-threatening MI. Specialized examinations such as intracoronary imaging [25] or ergonovine testing can facilitate the differential diagnosis, which can help to make proper treatment and guarantee better prognosis.

\section{Conclusions}

Physicians should be vigilant to adverse vascular events when treating patients who have been prescribed nilotinib. For MINOCA patients with a history of nilotinib treatment, an ergonovine provocation test should be done to check for the presence of CAS induced MINOCA. Further studies are needed to clarify the mechanism and to find better management options.

\footnotetext{
Abbreviations

AMl: Acute myocardial infarction; CAS: Coronary artery spasm; CAG: Coronary angiography; CML: Chronic myeloid leukemia; MINOCA: Myocardial Ischemia with No Obstructive Coronary Artery Disease; RCA: Right coronary artery; TKI: Tyrosine kinase inhibitor.
}

\section{Acknowledgements}

Not applicable.

\section{Authors' contributions}

WWC and BBD wrote the first draft and corrected the manuscript, $K L, Z X Y$ and XTW collected the data. BBD and PY revised the manuscript and figures. All authors read and approved the final manuscript.

\section{Funding}

This work was supported by the National Natural Science Foundation of China, No. 82100337; Industrial Grants, No. 2016220101000638. Both funding played a role in the design of the study, collection, analysis, and interpretation of the data and in writing the manuscript.

\section{Availability of data and materials}

The data/figures used and/or analyzed in this case are available from the corresponding author upon reasonable request.

\section{Declarations}

Ethics approval and consent to participate

The patient provided informed consent to participate.

\section{Consent to publication}

Written informed consent was obtained from the patient for the publication of this case report and any accompanying images.

\section{Competing interests}

The authors declare that they have no competing interests.

\section{Author details}

${ }^{1}$ Department of Cardiology, China-Japan Union Hospital of Jilin University, Xiantai, Street No. 126, Changchun 130033, Jilin Province, China. ${ }^{2}$ Jilin Provincial Cardiovascular Research Institute, Jilin Provincial Engineering Laboratory for Endothelial Function and Genetic Diagnosis of Cardiovascular Disease, Changchun 130031, Jilin Province, China. ${ }^{3}$ Department of Hematology, The First Hospital of Jilin University, Jilin Provincial Hematology Research Institute, National Key Discipline in Hematology and Oncology, Changchun 130021, Jilin Province, China.

Received: 17 October 2021 Accepted: 9 February 2022 Published online: 13 February 2022

\section{References}

1. Thygesen K, Alpert JS, Jaffe AS, Chaitman BR, Bax JJ, Morrow DA, White HD, Thygesen K, Alpert JS, Jaffe AS, et al. Fourth universal definition of myocardial infarction (2018). Eur Heart J. 2019:40(3):237-69.

2. Tamis-Holland JE, Jneid H, Reynolds HR, Agewall S, Brilakis ES, Brown TM, Lerman A, Cushman M, Kumbhani DJ, Arslanian-Engoren C, et al. Contemporary diagnosis and management of patients with myocardial infarction in the absence of obstructive coronary artery disease: a scientific statement from the American Heart Association. Circulation. 2019;139(18):e891-908.

3. Du B-B, Wang X-T, Tong Y-L, Liu K, Li P-P, Li X-D, Yang P, Wang Y. Optical coherence tomography guided treatment avoids stenting in an antiphospholipid syndrome patient: a case report. World J Clin Cases. 2020;8(11):2399-405

4. Chen W, Yu Z, Li S, Wagatsuma K, Du B, Yang P. Concomitant acute myocardial infarction and acute pulmonary embolism caused by paradoxical embolism: a case report. BMC Cardiovasc Disord. 2021;21(1):313.

5. Sacha T, Saglio G. Nilotinib in the treatment of chronic myeloid leukemia. Future Oncol (London, England). 2019;15(9):953-65.

6. Manouchehri A, Kanu E, Mauro MJ, Aday AW, Lindner JR, Moslehi J. Tyrosine kinase inhibitors in leukemia and cardiovascular events. Arterioscler Thromb Vasc Biol. 2020;40(2):301-8.

7. Aichberger KJ, Herndlhofer S, Schernthaner GH, Schillinger M, Mitterbauer-Hohendanner G, Sillaber C, Valent P. Progressive peripheral arterial occlusive disease and other vascular events during nilotinib therapy in CML. Am J Hematol. 2011:86(7):533-9.

8. Scalone G, Niccoli G, Crea F. Editor's Choice- Pathophysiology, diagnosis and management of MINOCA: an update. Eur Heart J Acute CardiovasC Care. 2019;8(1):54-62

9. Minson AG, Cummins K, Fox L, Costello B, Yeung D, Cleary R, Forsyth C, Tatarczuch M, Burbury K, Motorna O, et al. The natural history of vascular and other complications in patients treated with nilotinib for chronic myeloid leukemia. Blood Adv. 2019;3(7):1084-91.

10. Jain P, Kantarjian H, Boddu PC, Nogueras-González GM, Verstovsek S, Garcia-Manero G, Borthakur G, Sasaki K, Kadia TM, Sam P, et al. Analysis of cardiovascular and arteriothrombotic adverse events in chronic-phase CML patients after frontline TKIs. Blood Adv. 2019;3(6):851-61. 
11. Chen MT, Huang ST, Lin CW, Ko BS, Chen WJ, Huang HH, Hsiao FY. Tyrosine kinase inhibitors and vascular adverse events in patients with chronic myeloid leukemia: a population-based, propensity score-matched cohort study. Oncologist. 2021;26(11):974-82.

12. Coon EA, Zalewski NL, Hoffman EM, Tefferi A, Flemming KD. Nilotinib treatment-associated cerebrovascular disease and stroke. Am J Hematol. 2013;88(6):534-5.

13. Maurizot A, Beressi JP, Manéglier B, de la Marre NH, Spentchian M, Soury P, Solvet-Sebire P, Collet-Gaudillat C, Baud JM, Livarek B, et al. Rapid clinical improvement of peripheral artery occlusive disease symptoms after nilotinib discontinuation despite persisting vascular occlusion. Blood Cancer J. 2014;4(9):e247-e247.

14. Caocci G, Mulas O, Capodanno I, Bonifacio M, Annunziata M, Galimberti S, Luciano L, Tiribelli M, Martino B, Castagnetti F, et al. Low-density lipoprotein (LDL) levels and risk of arterial occlusive events in chronic myeloid leukemia patients treated with nilotinib. Ann Hematol. 2021;100(8):2005-14.

15. Omarjee $L$, Jaquinandi $V$, Mahe $G$. The use of the tyrosine kinase inhibitor Nilotinib in Spondyloarthritis: does targeting inflammatory pathways with a treatment lead to vascular toxicity? J Transl Med. 2017;15(1):255.

16. Roa-Chamorro R, Torres-Quintero L, García de Los Ríos C, Puerta-Puerta JM, González-Bustos P, Mediavilla-García JD. Progressive cardiovascular disease in a patient under treatment with nilotinib. Clinica e investigacion en arteriosclerosis: publicacion oficial de la Sociedad Espanola de Arteriosclerosis 2020, 32(2):66-69.

17. Fiets RB, Staal AHJ, Cramer GE, Blijlevens NMA. Coronary artery spasms due to tyrosine kinase inhibitors used in chronic myeloid leukemia. Neth J Med. 2018;76(7):330-5.

18. Maruta S, Usami K, Tajiri K, Otani M, Hiraya D, Watabe H, Hoshi T, Sato A, leda M. Vasospastic angina in a chronic myeloid leukemia patient treated with nilotinib. Cardiooncology. 2021;7(1):31.

19. Aghel N, Lipton JH, Atenafu EG, Kim DDH, Delgado DH. Cardiovascular events after exposure to nilotinib in chronic myeloid leukemia: long-term follow-up. Clin Lymphoma Myeloma Leuk. 2017;17(12):870-878.e871.

20. Caocci G, Mulas O, Annunziata M, Luciano L, Bonifacio M, Orlandi EM, Pregno P, Galimberti S, Russo Rossi A, Abruzzese E, et al. Cardiovascular toxicity in patients with chronic myeloid leukemia treated with second-generation tyrosine kinase inhibitors in the real-life practice: identification of risk factors and the role of prophylaxis. Am J Hematol. 2018;93(7):E159-e161.

21. Montone RA, Niccoli G, Fracassi F, Russo M, Gurgoglione F, Cammà G, Lanza GA, Crea F. Patients with acute myocardial infarction and nonobstructive coronary arteries: safety and prognostic relevance of invasive coronary provocative tests. Eur Heart J. 2018;39(2):91-8.

22. Slavich M, Patel RS. Coronary artery spasm: current knowledge and residual uncertainties. Int J Cardiol Heart Vasculature. 2016;10:47-53.

23. Chu G, Zhang G, Zhang Z, Liu S, Wen Q, Sun B. Clinical outcome of coronary stenting in patients with variant angina refractory to medical treatment: a consecutive single-center analysis. Med Princ Pract. 2013;22:583-7.

24. Beltrame JF, Crea F, Kaski JC, Ogawa H, Ong P, Sechtem U, Shimokawa H, Bairey Merz CN. International standardization of diagnostic criteria for vasospastic angina. Eur Heart J. 2017;38(33):2565-8.

25. Kiriyama H, Ishida J, Kadowaki H, Iwasa T, Kiyosue A, Kodera S, Ando J, Akazawa H, Komuro I. OCT-based management of nilotinib-associated CAD in a patient with chronic myeloid leukemia. JACC: CardioOncol. 2019, 1(2):318-321.

\section{Publisher's Note}

Springer Nature remains neutral with regard to jurisdictional claims in published maps and institutional affiliations.

Ready to submit your research? Choose BMC and benefit from:

- fast, convenient online submission

- thorough peer review by experienced researchers in your field

- rapid publication on acceptance

- support for research data, including large and complex data types

- gold Open Access which fosters wider collaboration and increased citations

- maximum visibility for your research: over 100M website views per year

At BMC, research is always in progress.

Learn more biomedcentral.com/submissions 